\title{
An A-C Resistance Thermometer Bridge
}

\author{
Robert D. Cutkosky \\ Institute for Basic Standards, National Bureau of Standards, Washington, D. C. 20234
}

(December 16, 1969)

\begin{abstract}
A $400 \mathrm{~Hz}$ bridge designed specifically for measuring the resistances of platinum thermometers is described. When used in conjunction with a conventional $25 \mathrm{ohm}$ thermometer, the instrument can resolve a resistance change of 2 micro-ohms or less, or about 20 microdegrees. It is believed to be in error by no more than 3 micro-ohms throughout the useful temperature range of a conventional thermometer. The instrument features an automatic phase angle balance, and extensive use is made of multistage transformers and operational amplifiers.
\end{abstract}

Key words: Automatic phase balance; bridge; multistage transformers; operational amplifiers, resistance bridge; resistance thermometry; temperature measurement.

\section{Introduction}

A number of new resistance thermometer bridges have been described in the last several years $[1,2,3,4] .{ }^{1}$ The incentive behind this rash of activity has been to take advantage of recent advances in bridge networks and null detectors, in order to provide a measurement accuracy closer to the limit set by thermal agitation noise in the thermometer than had been possible using the older technology. Most of the new resistance thermometer bridges, including the design described here, utilize alternating current rather than direct current, because of the resultant freedom from errors due to changing thermal emf's, and because of the availability of low noise a-c amplifiers. At the present time, inexpensive amplifiers with optimum noise figures smaller than one $\mathrm{dB}$ are only available for frequencies higher than a few hundred hertz. For this reason in part, the thermometer bridge described here was designed to operate at $400 \mathrm{~Hz}$.

Factors which were considered in the choice of the measuring frequency for the bridge were, in addition to amplifier noise, the existence of the technology involving precision decade inductive dividers and multistage transformers, and the technology involving operational amplifiers. Both technologies can be used to full advantage at $400 \mathrm{~Hz}$. Some problems might be expected due to the unavoidable frequency dependence of the resistance thermometer, but our measurements indicated that the resistance at the triple point of water of the particular thermometer used in our experiments did not change by more than 1 part per million (ppm) from dc to $400 \mathrm{~Hz}$. The frequency dependence of the thermometer is in any

\footnotetext{
${ }^{1}$ Figures in brackets indicate the literature references at the end of this paper.
}

case unimportant if it is both calibrated and used at the same frequency. It may, however, be necessary to select a standard frequency for use in resistance thermometry.

Early in the design of a resistance thermometer bridge it is necessary to choose a specific configuration for the leads and terminals to be used with the thermometer. The most reliable and most precisely defined lead arrangement available at this time is the so-called four-terminal-pair system [5], in which each of the four leads of the standard is provided with a coaxial shield.

We have elected to retain the usual four-terminal lead configuration, but modify our thermometers to accept a pair of coaxial cables. One cable constitutes the "current" terminals of the thermometer, and the other constitutes the "potential" terminals. This decision greatly simplifies the design of the bridge, and is acceptable in this case because the principal uncertainty introduced thereby is only in the phase angle of the resistor, which is of no importance in resistance thermometry.

A disadvantage of the two-pair system is that the cable admittances appear in parallel with the thermometer, but tests have shown that the shunt conductance of 15 meters of RG 58/U cable changes the apparent resistance at $400 \mathrm{~Hz}$ of a conventional $25 \Omega$ thermometer by less than two parts in $10^{8}$. The cable capacitance causes a substantial phase angle under these conditions, but this can be readily handled.

A key part of the circuit described below is a threestage transformer. A three-stage transformer is similar to the well known two-stage transformer $[6,7]$, but contains three high-permeability cores rather than two. The construction of a three-stage transformer begins with a 


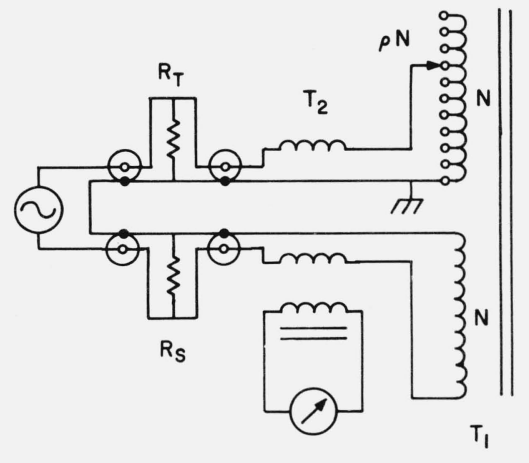

Figure 1. Elementary bridge circuit.

winding called the first stage winding on a single core. In a circuit diagram it is helpful to label this core "inside." The second core is then placed next to the completed first stage, and another winding called the second stage winding is wound around both cores. This assembly is a two-stage transformer. Following this, the third core is placed next to the two-stage transformer and the final winding, called the third stage winding, is wound around all three cores. The relative positions of the cores and windings can be duplicated on the circuit diagram starting with the labeled inside core. This provides a visual picture of the construction of the transformer, from which its operation can be easily inferred. A three-stage transformer is an obvious extension of a two-stage transformer, and its behavior can be calculated using the methods described in the publications cited above.

\section{Bridge Circuitry}

The general plan of the bridge is given in figure 1, which shows the resistance thermometer $R_{t}$ in series with a standard resistor $R_{s}$ and a current generator. A precision adjustable transformer $T_{1}$ of very high input impedance is connected to the potential leads of the two resistors via a three winding detector transformer, $T_{2}$. If the input impedance of $T_{1}$ is sufficiently high, the detector will register a null when $R_{t}=\rho R_{s}$, where $\rho$ is the ratio of $T_{1}$.

In order to maintain a high accuracy in the presence of lead and winding impedances which may be as large as several ohms, without the necessity for separately balancing these impedances, the input impedance of $T_{1}$ must be at least $10^{8} \Omega$. The easiest way to achieve this is by constructing $T_{1}$ as a two-stage transformer. The first stage of $T_{1}$ could then be excited by connecting it directly in parallel with the generator supplying current to $R_{t}$ and $R_{s}$, provided that the total number of turns were continuously adjusted to equal $N(1+\rho)$ by ganged switching. This method of excitation was rejected because we wanted to be able to use the bridge not only with $R_{s}=100 \Omega$ and a standard $25 \Omega$ resistance thermometer, but also with $R_{s}=0.1 \Omega$ and a special $0.025 \Omega$ thermometer designed for measurements at the gold point. In the latter case, the very large potential drops in the current leads of the resistors would have resulted in an incorrect excitation of the first stage of $T_{1}$.

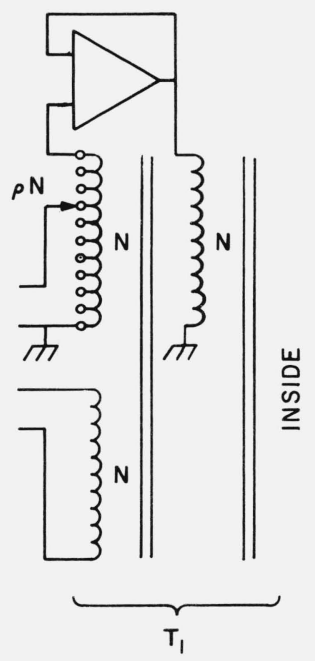

FIgURE 2. Excitation of a two-stage transformer with a voltage follower.

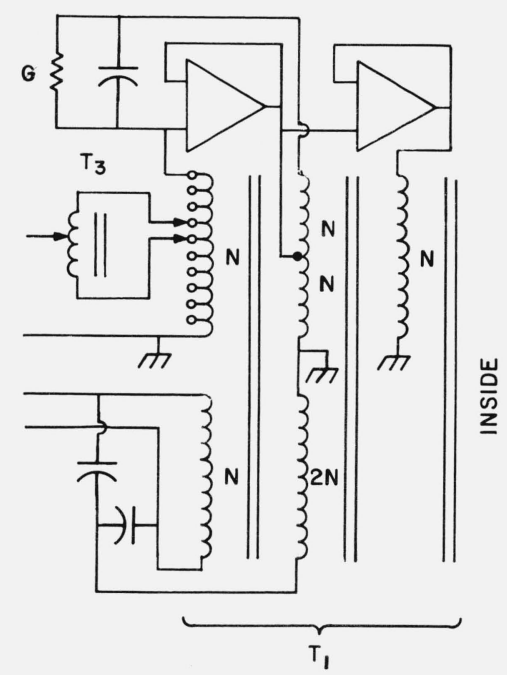

FigurE 3. Excitation of a three-stage transformer with two voltage followers.

The technique used for compensating the interwinding capacitance of the third stage is also shown.

Figure 2 shows how an operational amplifier connected as a voltage follower can be used to automatically supply the correct excitation to the first stage of a two-stage transformer, and yield a high input impedance as viewed at the second stage. This circuit is inherently highly stable, and is much less prone to oscillate than alternative circuits based on the permeability multiplier developed by D. L. H. Gibbings [8]. The actual circuit used for $T_{1}$ contains two voltage followers and a three-stage transformer as shown in figure 3. Additional windings were put on the second stage as shown to provide a means of compensating the interwinding capacitances of the third or outer stage. Small resistors (not shown) were added in series with the compensating capacitors to suppress high 


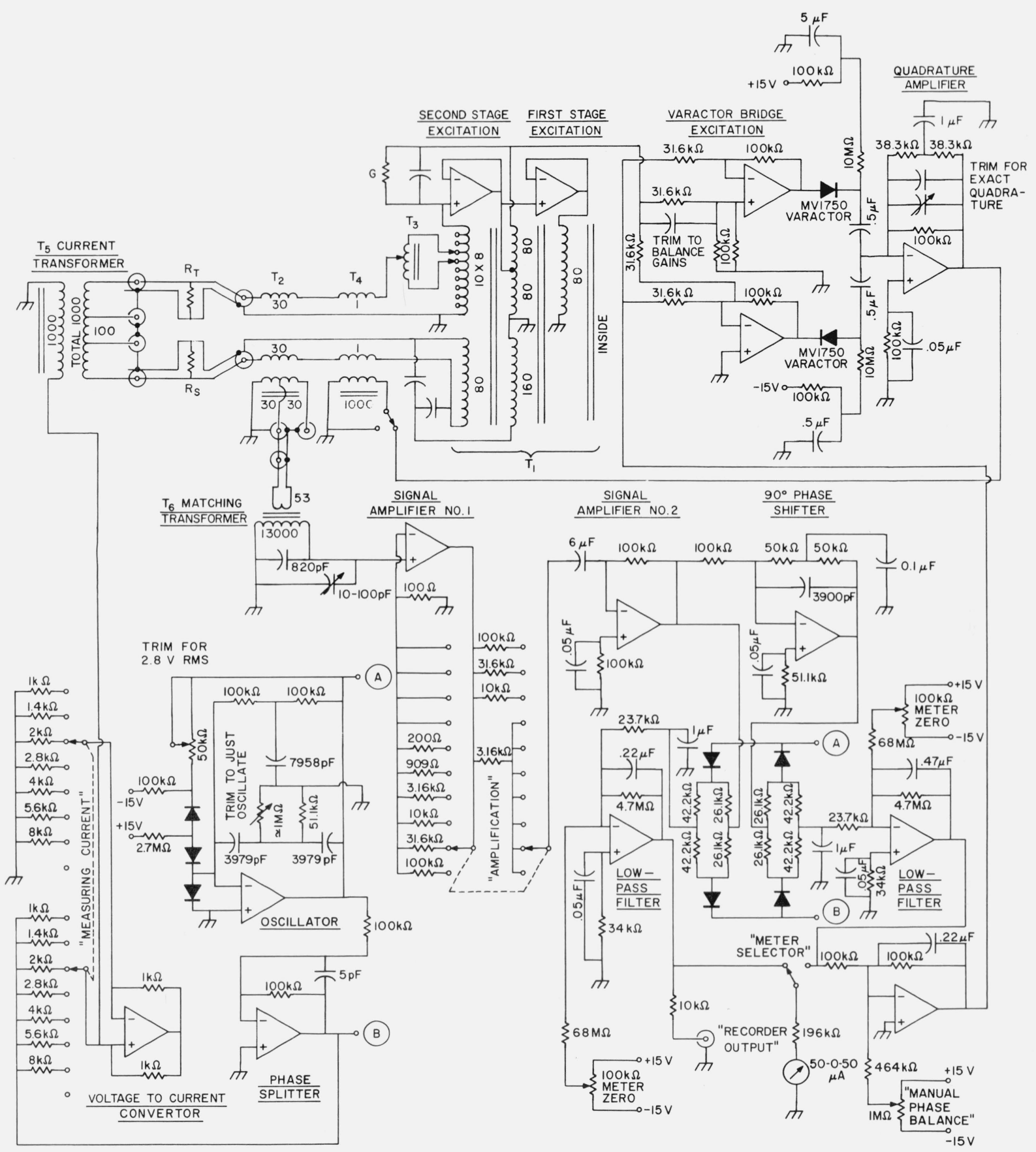

FIGURE 4. Complete circuit of the a-c resistance thermometer bridge.

The numbers associated with each transformer winding indicate the number of turns.

frequency oscillations. The third stage was constructed with polytetrafluoroethylene insulated wire to minimize the shunt conductance between the windings.

Only the first decade of $T_{1}$ was specially constructed, the lower seven decades being obtained from a commercial inductive divider $T_{3}$, which bridges adjacent taps on $T_{1}$. The equivalent shunt resistance of $T_{3}$ is about $10^{6} \Omega$, and its effect on the input impedance seen across a fixed winding on the third stage of $T_{1}$ is independent of $\rho$.
The conductance labeled $\mathrm{G}$ in figure 3 is selected to eliminate the potential lead current that would otherwise result. A resistor of about $10^{8} \Omega$ was required.

The detector system used with the bridge contains a matching transformer, an amplifier with an optimum source impedance of $6 \times 10^{6} \Omega$ and a noise figure of $0.2 \mathrm{~dB}$, and two phase-sensitive detectors followed by low pass filters. The first phase-sensitive detector is adjusted so that it responds to a resistive unbalance in the 
bridge. Its output is passed through a filter with a $1 \mathrm{~s}$ time constant, and displayed on a panel voltmeter and an optional external recorder. The second phase-sensitive detector responds to a phase angle unbalance. Its filtered output is used to control the level of a quadrature voltage added in series with the detector transformer, $T_{2}$, to provide an automatic phase angle balance.

The quadrature balance voltage was obtained by amplifying the output of the voltage follower driving the second stage of $T_{1}$, and applying this voltage to a bridge involving a pair of varactor diodes. The d-c output of the second phase-sensitive detector determines the biases on the two varactor diodes, and hence controls the short circuit current at the varactor bridge detector junction. This current is $90^{\circ}$ out of phase with the input voltage, since the varactor diodes behave like capacitors. An operational amplifier connected as a current to voltage converter then produces an output voltage proportional to this a-c current, and the voltage is inserted in series with $T_{2}$. A very similar automatic phase balance system using a balanced mutual inductor rather than varactor diodes has been developed by A. M. Thompson [4].

The behavior of the feedback loop sketched above is somewhat difficult to calculate, since the effective loop gain depends not only upon the gains of a series of a-c and d-c amplifiers, but also upon the excitation current applied to the resistance bridge. The bandwidth of the feedback loop is limited principally by the low pass filter following the second phase-sensitive detector, but also by the finite bandwidth of the tuned matching transformer preceding the detector amplifier. In order to avoid low frequency instabilities due to excessive phase shifts, the $\mathrm{Q}$ of the matching transformer must be kept small.

The complete circuit of the resistance thermometer bridge is shown in figure 4. In addition to the features described above, it includes a constant current generator with switch-selectable output currents to excite the bridge, so that the measuring current is independent of the temperature of the thermometer. Provisions are also made for changing the ratio of the current transformer driving the bridge and the ratio of the detector matching transformer to accommodate either a $25 \Omega$ or a $0.025 \Omega$ resistance thermometer. A liberal use of mu-metal shielding was required, not only to avoid interactions between various parts of the bridge, but also to prevent saturation of the detector by harmonics of the $60 \mathrm{~Hz}$ power supply.

\section{Performance}

When $R_{s}$ is a $100 \Omega$ standard, a change of one step in the last decade of the bridge corresponds to a resistance change of $1 \mu \Omega$, or about $10 \mu \mathrm{deg}$ when $R_{t}$ is a conventional $25 \Omega$ platinum thermometer. With a measuring current of $1 \mathrm{~mA}$, a bridge unbalance of $2 \mu \Omega$ can be resolved throughout the useful temperature range of the thermometer. Tests have been made which indicate that the bridge reading is in error by no more than $3 \mu \Omega$ over the entire range of 0 to $100 \Omega$, provided that the RG 58/U thermometer cables are no longer than $15 \mathrm{~m}$.

When $R_{s}$ is a $0.1 \Omega$ standard and $R_{t}$ is a $0.025 \Omega$ platinum thermometer, and with a measuring current of $0.028 \mathrm{~A}$, the bridge will resolve a temperature change of $100 \mu \mathrm{deg}$. This relatively large uncertainty greatly exceeds that which would be produced by thermal agitation noise in the thermometer, and is caused principally by thermal agitation noise in the winding resistance of the commercial inductive divider, $T_{3}$. For the time being, a resolution of $100 \mu \mathrm{deg}$ is adequate for the measurements to be made at the gold point.

The accurate calibration of the bridge was greatly simplified through the use of a special two-stage inductive divider constructed and made available by Wilbur C. Sze of the NBS Electricity Division, High Voltage Section. The reliability of the bridge was verified through an exhaustive series of measurements made by John L. Riddle and William R. Bigge of the NBS Heat Division, Temperature Section. The special $0.1 \Omega$ standard resistor for use with a thermometer designed for operation at the gold point was constructed of a gold-chromium alloy by James L. Thomas while serving as a consultant to the NBS Electricity Division. The $0.025 \Omega$ platinum thermometer is currently under development by L. A. Guildner and R. L. Anderson of the NBS Heat Division, Temperature Section, who provided much of the stimulus for the work reported here.

\section{References}

[1] Hill, J. J., and Miller, A. P., An A. C. double bridge with inductively coupled ratio arms for precision platinum resistance thermometry, Proc. I.E.E. 110, No. 2 (1963).

[2] Automatic Systems Laboratories, Ltd., Precision Resistance Bridges (Brochure) Leighton Buzzard, Beds., England.

[3] Kusters, N. L., and MacMartin, M. P., National Research Council, Ottawa, Canada (private communication).

[4] Thompson, A. M., National Science Laboratory, Sydney, Australia (private communication).

[5] Cutkosky, R. D., Four-Terminal-Pair Networks as Precision Admittance and Impedance Standards, Communication and Electronics, 70 (Jan. 1964).

[6] Brooks, H. B., and Holtz, F. C., The Two-Stage Current Transformer, AIEE Trans. 41, 383 (1922).

[7] Cutkosky, R. D., Active and Passive Direct Reading Ratio Sets for the Comparison of Audio Frequency Admittances, Nat. Bur. Stand. (U.S.), 68C (Eng. and Instr.) No. 4, 195-322 (Oct.-Dec. 1964).

[8] Gibbings, D. L. H., A Circuit for Reducing the Excitation Current of Inductive Devise, Proc. I.E.E. 108B, 339-343 (1961).

(Paper 74C1\&2-295) 